\title{
Genetic diversity of canine gastric helicobacters, Helicobacter bizzozeronii and $H$. salomonis studied by pulsed-field gel electrophoresis
}

\author{
M-L. HÄNNINEN and U. HIRVI \\ Faculty of Veterinary Medicine, Department of Food and Environmental Hygiene, PO Box 57, FIN-00014 \\ Helsinki University, Helsinki, Finland
}

\begin{abstract}
Genetic diversity of Helicobacter bizzozeronii and $\boldsymbol{H}$. salomonis, two recently identified canine gastric Helicobacter spp., was studied by pulsed-field gel electrophoresis (PFGE). All 15 Finnish $H$. bizzozeronii strains collected between 1991 and 1996 from pet dogs produced different PFGE patterns with all restriction endonucleases studied (AscI, ApaI, SpeI, NotI and Pacl) suggesting significant genetic diversity. The five independent $H$. salomonis strains produced four different patterns with these enzymes; two strains showed identical patterns with all the enzymes. Three separate isolates from one dog had identical patterns, suggesting long-lasting infection with the same strain. $H$. salomonis strains had several small fragments common for all strains, suggesting relatedness. The PFGE method was shown to be useful for epidemiological studies of canine gastric helicobacter infection. Hybridisation of the DNA digests with digoxigeninlabelled ureB or 16S rRNA gene probes generated by PCR indicated conservation in the localisation of these genes in the $H$. salomonis genome, because the probes hybridised with similar size fragments of different strains. In contrast, the probes hybridised with different size fragments of $\mathrm{H}$. bizzozeronii strains. Comparison of Southern blots of PFGE patterns digested with SpeI, ApaI and $A s c I$ indicated that each species has two 16S rRNA genes and one urease gene. Genome sizes of $11 \mathrm{H}$. bizzozeronii strains estimated from SpeI and NotI patterns were c. 1.6-1.9 Mb and those of five $H$. salomonis strains estimated from NotI and PacI patterns were $c$. 1.7-1.8 Mb.
\end{abstract}

\section{Introduction}

The gastric mucosa of dogs is colonised by three Helicobacter spp. - H. felis [1], H. bizzozeronii [2] and H. salomonis [3]. Some dogs are colonised also by an uncharacterised Helicobacter sp., provisionally called 'flexispiras' [4]. Helicobacter infection of canine gastric mucosa is common, almost all dogs being infected with one or more gastric Helicobacter spp. [4-6]. The pathogenicity of these organisms is unknown. They are fastidious and their in-vitro culture requires special conditions [2].

H. pylori, a human-adapted Helicobacter sp., has been well-characterised because of its significance in the pathogenesis of chronic active gastritis and peptic ulcer. This organism is also strongly associated with human gastric cancer [7]. The genome of $H$. pylori has been characterised and the whole genome

Received 17 April 1998; accepted 23 June 1998.

Corresponding author: Dr M-L. Hänninen. sequence analysis has been published recently [8]. The intra-species genetic diversity has been studied also by various genetic methods such as macrorestriction analysis of the whole genome by pulsedfield gel electrophoresis (PFGE) $[9,10]$, by multi-locus enzyme electrophoresis (MLEE) [11], ribotyping [12], randomly amplified polymorphic DNA [13] and polymorphism of urease $[14,15]$ and $v a c A[16]$ genes. All these methods have revealed significant genetic diversity, probably a necessary characteristic for a pathogen adapted to one mammal species, man. Genetic diversity of other Helicobacter spp. is not well known, except for the genome of $H$. mustelae, which was shown to be rather homogeneous by PFGE, when American and European strains were compared [17], whereas the PFGE patterns of $H$. hepaticus showed some diversity [18].

In the present study, genetic diversity of $H$. bizzozeronii and $H$. salomonis strains from Finnish dogs was studied by PFGE with several restriction endonucleases. PFGE patterns allowed the determination of 
the genome sizes and the number of ure B and $16 \mathrm{~S}$ rRNA genes.

\section{Materials and methods}

\section{Bacterial strains}

H. bizzozeronii strains were isolated from 15 Finnish dogs between 1991 and 1996, and the $10 \mathrm{H}$. salomonis isolates came from Finnish dogs between 1993 and 1996. Three of the $H$. salomonis isolates were cultured from one $\operatorname{dog}$ ( $\operatorname{dog}$ HS 8 , isolates HS 8a, HS 8b, HS $8 \mathrm{c}$ ) on separate occasions over a period of c. 2 years, and one of the isolates originated from a puppy of $c .5$ months of age when it had been living with the puppies of dog HS 8 (isolate HS 8d). Both Helicobacter spp. have already been characterised by phenotypic testing, SDS-PAGE, dot-blot and reassociation DNA-DNA hybridisation and 16S rRNA gene sequencing $[2,3]$. The organisms were stored at $-70^{\circ} \mathrm{C}$, and most of the strains were subcultured 10-20 times after their first isolation.

\section{DNA preparation and digestion for PFGE}

The organisms were grown on three-to-six Brucella blood agar plates with antibiotics in a micro-aerobic atmosphere for 2-3 days [2]. Bacterial cells were collected in Brucella broth (Oxoid), pelleted, suspended in PBS and treated with formaldehyde to inactivate endogeneous nucleases [19]. The cells were centrifuged (1800 rpm) for $10 \mathrm{~min}$ at $4^{\circ} \mathrm{C}$ and washed in PBS, and DNA in agar plugs for PFGE was prepared as described previously [20]. Several restriction endonucleases (AvrI, Asc II, Apa I, Cla I, EcoRI, MluI, Not I, $P a c$ I, PvuII, Spe I and $S f i \mathrm{I})$ were tested to find the optimal digestion for genetic diversity studies and genome size determination. DNA digests were prepared according to the instructions of the restriction endonuclease manufacturers (Boehringer Mannheim, Mannheim, Germany and New England Biolabs, Beverly, MA, USA).

\section{PFGE}

PFGE of digested genomic DNA was performed in agarose $1 \%$ gels in $0.5 \times$ TBE $(4.45 \mathrm{mM}$ Tris, $4.45 \mathrm{mM}$ boric acid, $0.1 \mathrm{mM}$ EDTA) with Gene Navigator electrophoresis equipment (Pharmacia LKB Biotechnology AB, Uppsala, Sweden). Depending on the fragment sizes, different pulse and electrophoresis run times were used. Genome sizes were estimated from Not I digests after five different programmes with a ramped pulse, starting with intial pulses from 2 to $20 \mathrm{~s}$ and with final pulses from 40 to $130 \mathrm{~s}$, with running times from 18 to $22 \mathrm{~h}$. For genome size determination from SpeI digests, three electrophoresis run programmes were applied: a ramped pulse from 2 to $25 \mathrm{~s} / 18 \mathrm{~h}$; a stepping programme $(0.5 \mathrm{~s} / 1 \mathrm{~h} ; 0.7 \mathrm{~s} / 1 \mathrm{~h}$; $0.9 \mathrm{~s} / 1 \mathrm{~h} ; 2 \mathrm{~s} / 3 \mathrm{~h} ; 4 \mathrm{~s} / 4 \mathrm{~h}$ and $6 \mathrm{~s} / 4 \mathrm{~h})$; and a short programme for small fragments of $<15 \mathrm{~kb}$, with an initial pulse of $0.5 \mathrm{~s}$ and a final pulse of $8 \mathrm{~s}$ for $8 \mathrm{~h}$. Different appropriate fragment size markers were used: chromosomal DNA of Saccharomyces cerevisiae (range $225-2500 \mathrm{~kb}$ ), bacteriophage- $\lambda$ ladders starting from $15 \mathrm{~kb}$ (range $15-300 \mathrm{~kb}$ ) or $48.5 \mathrm{~kb}$ (range $50-$ $1000 \mathrm{~kb})$ and a low-range marker $(0.1-200 \mathrm{~kb})$. All size markers were from New England Biolabs. After electrophoresis, the gels were stained with ethidium bromide $5 \%$, exposed to UV light and photographed.

\section{Genome sizes}

Genome sizes were estimated by measuring the migration distances of individual fragments and referring these to a standard curve of migration distances plotted against log mol.wt of DNA size standards. Genome sizes were obtained by adding the sizes of all the individual fragments.

\section{Southern blotting and DNA hybridisation}

DNA from an agarose gel was transferred to a nylon membrane (Micron Separations, Westborough, MA, USA) by Vacu Gene XL, a vacuum blotting apparatus (Pharmacia LKB Biotechnology LKB, Sollentuna, Sweden). After denaturation of DNA under UV light, the membrane was pre-hybridised at $58^{\circ} \mathrm{C}$ for $2 \mathrm{~h}$ and hybridised at $58^{\circ} \mathrm{C}$ with digoxigenin-labelled ure $\mathrm{B}$ or $16 \mathrm{~S}$ rRNA gene probes for $16 \mathrm{~h}$. After hybridisation, the membrane was washed twice for $5 \mathrm{~min}$ each in $2 \times \mathrm{SSC}(1 \times \mathrm{SSC}$ contained $0.15 \mathrm{M} \mathrm{NaCl}, 0.015 \mathrm{M}$ sodium citrate and SDS $0.1 \%$ ) at room temperature and then twice at $58^{\circ} \mathrm{C}(15 \mathrm{~min}$ each). The hybridised fragments were visualised colorimetrically with antidigoxigenin-alkaline phosphatase, nitro-blue tetrazolium chloride and 5-bromo-4-chloro-3-indolyl phosphate 4-toluidine salt as substrates (Boehringer Mannheim).

\section{Preparation of probes from $P C R$ products of $16 S$} $r R N A$ and ureB gene fragments

Helicobacter genus-specific primers, H276f (5'CTATGACGGGTATCCGGC3') and H676r (5'ATTCCACCTACCTCTCCCA3') were used for amplification of a 374-bp fragment from the 16S rDNA gene of $H$. bizzozeronii CGUG 35545 [21], and a nested PCR was applied to amplify first a $1-\mathrm{kb}$ ure B fragment and then an 800-bp fragment from DNA of $H$. bizzozeronii CGUG 35545. The primers were from H. pylori and ' $H$. heilmannii' urease genes, and the PCR amplification programme was as described by Solnick et al. [22]. The reaction products were electrophoresed in an ID agarose $2 \%$ gel (FMC BioProducts, Rockland, USA) and visualised after ethidium bromide staining under UV light. Both PCR products were labelled by random priming with digoxigenin-dUTP according to the manufacturer's instructions for the DNA labelling and detection kit (Boehringer Mannheim). 


\section{Results}

\section{Restriction endonucleases}

Among restriction endonucleases tested, SpeI (Fig. 1), $A s c \mathrm{I}$ and Apa I produced c. 20 fragments of c. 10$500 \mathrm{~kb}$ for $H$. bizzozeronii. The DNA of $H$. salomonis was digested with these enzymes, and many small fragments were produced. Examples of the patterns of Spe I digests of $H$. salomonis are presented in Fig. 2 which shows a number of small fragments $<15 \mathrm{~kb}$. Not I produced three to nine fragments from the DNA of each species with sizes from 40 to $1000 \mathrm{~kb}$ (Fig. 3, lanes $1-8$ ). DNA of all $H$. salomonis strains was cut with $N o t$ I, but DNA of most strains of $H$. bizzozeronii was not digested with this enzyme. Sfi I produced $c .10$ fragments from $H$. salomonis DNA; however, $H$. bizzozeronii strains were not digested with this enzyme (Fig. 3, lanes 9-13). PacI digested DNA from $H$. salomonis and $H$. bizzozeronii strains but much of the DNA was only partially digested, especially from $H$. bizzozeronii. Other endonucleases tested produced a fragment pattern dominated by a great number of small fragments, so these enzymes were not used for further studies. The patterns produced were stable for digests done from the same plugs over a period of several months or from different plugs prepared after several subcultures.

\section{Genetic heterogeneity}

All $H$. bizzozeronii strains produced different restriction patterns with all endonucleases. An example of DNA digested with Spe I is shown in Fig. 1. No $\begin{array}{lllllllll}1 & 2 & 3 & 4 & 5 & 6 & 7 & \mathrm{mw}\end{array}$

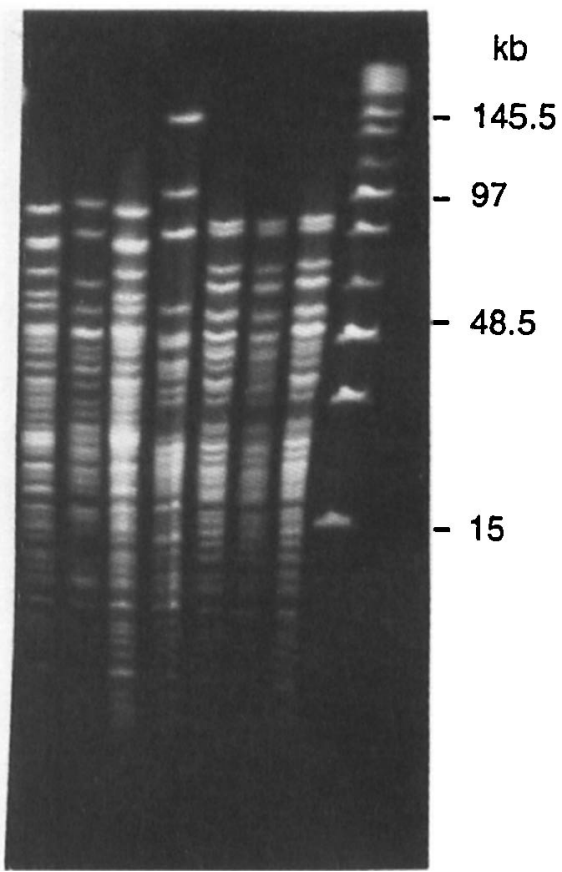

Fig. 2. PFGE patterns of seven $H$. salomonis isolates digested with SpeI and electrophoresed with the same stepping programme as in Fig. 1. Lane 1, HS 6; lane 2, HS 2; lane 3, HS 5 (pattern identical to lane 1); 4, HS 4 (CCUG 37845); 5, HS 8d (CCUG 37848); 6, HS 8b; 7, HS 1. Molecular size marker $\lambda$ concatamer $(15 \mathrm{~kb})$ and the sizes are indicated on the right.

fragments common to all strains were identified. Not I digested DNA from only 5 of $15 \mathrm{H}$. bizzozeronii strains (examples in Fig. 3, lanes 5-8).

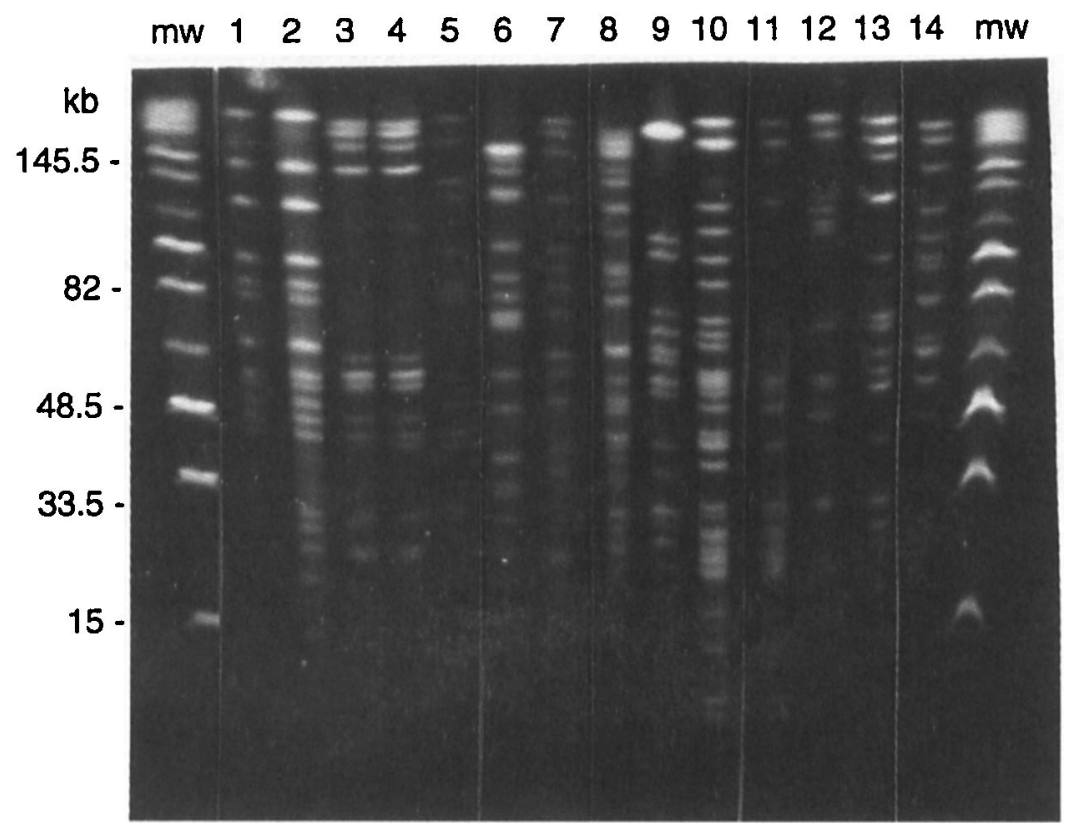

Fig. 1. Examples of SpeI-digested DNA from $H$. bizzozeronii, showing small and middle sized fragments $(<15 \mathrm{~kb}-$ $150 \mathrm{~kb}$ ). A stepping programme was used: $0.5 \mathrm{~s} / 1 \mathrm{~h} ; 0.7 \mathrm{~s} / 1 \mathrm{~h} ; 0.9 \mathrm{~s} / 1 \mathrm{~h} ; 2 \mathrm{~s} / 3 \mathrm{~h} ; 4 \mathrm{~s} / 4 \mathrm{~h}$ and $6 \mathrm{~s} / 4 \mathrm{~h}$. Lanes 1 and 2 strain CGUG 35545 (HB 1 isolates from 1994 and 1996); $\mathbf{3}$ and 4, two isolates of HB 6 from 1995 and 1996; 5, strain CGUG 35046 (HB 2); 6, strain HB 16; 7, strain HB 17; 8, strain HB 12; 9, strain HB 3; 10, strain HB 10; 11, strain HB $8 ; 12$, strain HB $14 ; 13$, strain HB $13 ; 14$, strain HB 9; mw, molecular size marker $\lambda$ concatamer (15 kb). Molecular sizes are indicated on the left. 


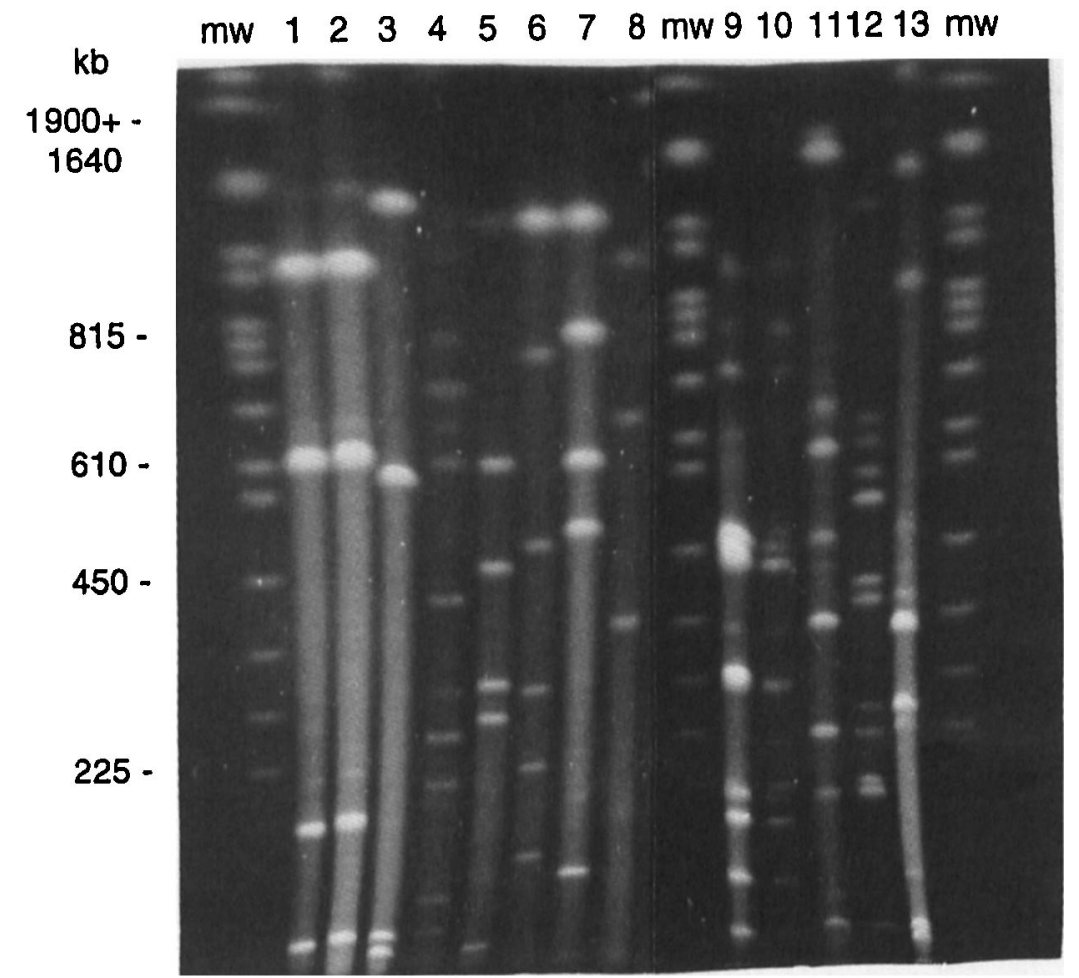

Fig. 3. PFGE patterns of Not I-digested (lanes 1-8) or Sfi I-digested (lanes 9-13) DNA from H. salomonis (HS) and $H$. bizzozeronii (HB). Lane 1, HS 6; 2, HS 5; 3, HS 4 (CGUG 37845); 4, HS 3; 5, HB 8; 6, HB 14; 7, HB 10; 8, HB 4; 9 , HS 8d (CCUG 37848); 10, HS 8b; 11, HS 6; 12, HS 2; 13, HS 4 CCUG 37845); molecular size marker yeast chromosome PFG marker $(\mathrm{kb})$.

Asc I, ApaI, SpeI (Fig. 2), NotI (Fig. 3, lanes 1-4), $S f i$ (Fig. 3, lanes 9-13) and PacI produced patterns useful for study of the genetic diversity of $H$. salomonis. These endonucleases digested all the isolates. In this group, three isolates from one dog (dog HS 8, isolates $8 \mathrm{a}, 8 \mathrm{~b}$ and $8 \mathrm{c}$ ) and from one puppy (isolate HS 8d) had identical PFGE patterns with all the enzymes. The SpeI patterns for the isolates HS $8 \mathrm{~d}$ and $\mathrm{HS} 8 \mathrm{~b}$ are shown in Fig. 2 (lanes 5 and 6) and the Not I patterns for the same isolates are shown in Fig. 3 (lanes 9 and 10). The H. salomonis strain from one dog (HS 1) had a PFGE pattern identical to the isolates from dog HS 8 and the puppy HS 8d with SpeI (Fig. 2, lane 7) and PacI; however, when Not I or Asc I was used for digestion, the patterns differed. Two other strains (HS 5 and HS 6) with no known association had identical patterns with all the endonucleases (SpeI, Asc I, NotI, PacI) (Fig. 2, lanes 1 and 3).

\section{Genome size}

H. bizzozeronii. Genome sizes for $11 \mathrm{H}$. bizzozeronii strains were estimated from PFGE patterns (Table 1). The sizes for five strains were estimated from NotI and SpeI digests and, for six strains, the estimation was performed with SpeI digest patterns only, because Not I did not digest the DNA of these strains. The fragments produced by other endonucleases either had too many small fragments ( $<15 \mathrm{~kb}$ (e.g., Apa I and AscI) or DNA cutting was unreliable due to partial digestion. Genome size estimation was performed with three to five different gels electrophoresed under different run programmes to determine the sizes of small, middle-size and large fragments. Genome sizes from Not $\mathrm{I}$ digests of five H. bizzozeronii strains varied from 1630 SD 22 to 1937 SD $21 \mathrm{~kb}$, while those from SpeI digests of 11 strains varied from 1558 SD 32 to 1945 SD $35 \mathrm{~kb}$ (Table 1). Similar sizes were estimated from Not I and SpeI digests. Fragments from Not I digests were fewer (2-4) than from SpeI digests (14-25).

H. salomonis. Genome sizes of five $H$. salomonis strains were estimated from NotI digests (Fig. 2) after five electrophoresis runs (Table 1). Spe I (Fig. 2), Apa I and Asc I digests were not used for genome size estimation because they produced too many small fragments $(<15 \mathrm{~kb})$. PacI digests were used for estimation of the sizes of the genomes of three strains. The genome sizes from Not I digests varied from 1675 SD 35 to 1805 SD $25 \mathrm{~kb}$ and from PacI digests from 1769 SD 19 to 1810 SD $28 \mathrm{~kb}$ (Table 1). Genome sizes of individual strains were similar from Not $\mathrm{I}$ and Pac I digests (Table 1).

\section{ureB and $16 S$ rRNA genes}

The ure B gene probe was hybridised with $A s c \mathrm{I}$ and Spe I digests. The number of fragments which hybridised with ureB was one or two, suggesting one urease gene for both Helicobacter spp. In $\mathrm{H}$. bizzozeronii digests, the ureB probe always hybridised with differ- 
Table 1. Genome sizes of $11 H$. bizzozeronii and five $H$. salomonis strains determined from PFGE patterns of Not I, SpeI or Pac I digests

\begin{tabular}{|c|c|c|c|}
\hline \multirow[b]{2}{*}{ Strain no. } & \multicolumn{3}{|c|}{ Genome size, kb, and (number of fragments) } \\
\hline & Not $\mathrm{I}$ & Spe I & PacI \\
\hline \multicolumn{4}{|l|}{ H. bizzozeronii } \\
\hline CCUG 35545 (HB 1) & Not digested & 1766 SD $24(18)$ & $\cdots$ \\
\hline CCUG 35046 (HB 2) & 1937 SD 21 & 1945 SD 35 (16) & ... \\
\hline HB 6 & 1745 SD $13(2)$ & 1770 SD $23(15)$ & $\cdots$ \\
\hline HB 5 & 1630 SD $22(3)$ & $1688 \mathrm{SD} 34(17)$ & $\cdots$ \\
\hline HB 10 & 1797 SD 18 (3) & 1795 SD $22(25)$ & $\cdots$ \\
\hline HB 14 & 1780 SD 15 (4) & Not done & $\cdots$ \\
\hline HB 17 & Not digested & 1706 SD $16(18)$ & $\ldots$ \\
\hline $\mathrm{HB} 16$ & Not digested & 1595 SD $21(18)$ & $\cdots$ \\
\hline HB 9 & Not digested & $1570 \mathrm{SD} 80$ (14) & $\cdots$ \\
\hline HB 13 & Not digested & 1558 SD 32 (14) & $\cdots$ \\
\hline HB 3 & Not digested & 1804 SD $27(18)$ & $\cdots$ \\
\hline \multicolumn{4}{|l|}{ H. salomonis } \\
\hline CCUG 37848 (HS 8d) & 1805 SD 25 (4) & $\cdots$ & $1796 \mathrm{SD} 16(12)^{*}$ \\
\hline CCUG 37845 (HS 4) & 1679 SD $25(6)$ & $\cdots$ & Not done \\
\hline HS 6 & 1752 SD $30(\cdots)$ & $\cdots$ & 1810 SD 28 \\
\hline HS 1 & 1799 SD $27(6)$ & $\cdots$ & 1769 SD $19(12)^{*}$ \\
\hline HS 3 & 1675 SD 35 (4) & $\cdots$ & Not done \\
\hline
\end{tabular}

${ }^{*}$ PacI patterns were identical.

ent fragments of different strains (results not shown). In contrast, the probe always hybridised with the same fragments of $H$. salomonis Asc I or SpeI DNA digests (Fig. 4).

The 16S rRNA probe hybridised with two to four fragments of AscI and ApaI digests of both $H$. bizzozeronii and $H$. salomonis, suggesting that these species have two copies of 16S rRNA genes. The probe always hybridised with the same fragments of $H$. salomonis strains, but with different fragments of H. bizzozeronii strains (Fig. 4).

\section{Discussion}

$H$. bizzozeronii and $H$. salomonis are recently described canine gastric helicobacters $[2,3]$ and their genetic diversity has not been studied previously. The present study showed that these two species may differ in the level of diversity, although the number of strains studied was limited because strains have been isolated only in Finland. All H. bizzozeronii strains isolated within the last several years from pet dogs had different PFGE patterns revealing significant genetic diversity. Unassociated $H$. salomonis strains isolated either from pet or experimental dogs also showed diversity in their PFGE patterns, but some common fragments were noted in Spe I, PacI, Sfi I and NotI digests. All three isolates from one $\mathrm{dog}$ and an isolate from a related puppy were identical, suggesting that the method produces stable patterns useful for studies of the epidemiology of canine gastric helicobacter infections. The dog was infected with same strain for the whole study period, $>2$ years.

The genome of $H$. pylori has shown significant diversity by all methods used and it has been regarded as one of the most heterogeneous species known [10]. The genomes of other Helicobacter spp. are not well described. In studies by Saunders et al. [18], $H$. muridarum strains from the USA and Europe showed

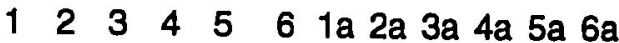

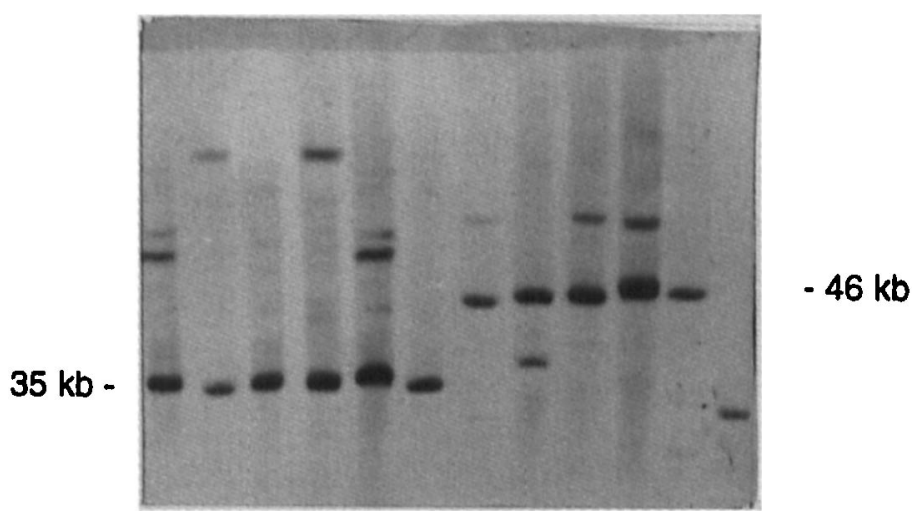

Fig. 4. Southern blotting of AscI (lanes 1-6) and SpeI (lanes 1a-6a) digests of $H$. salomonis probed with digoxigeninlabelled ure $\mathrm{B}$ gene probe. The molecular sizes of hybridised fragments are shown by an arrow. 
different PFGE patterns, suggesting genetic diversity. In contrast, in the PFGE analysis of a limited number of strains, the genetic diversity of $H$. mustelae was low [17]. It has been suggested that the significant genetic diversity of $H$. pylori strains reflects a long evolutionary history as a human pathogen $[10,13,18]$. Recently, the only recognised host for $H$. bizzozeronii and $H$. salomonis has been dogs $[2,3]$. In dogs, mixed infections have been noted with $H$. bizzozeronii and $H$. felis $[4,23]$, unlike $H$. pylori colonisation in human gastric mucosa [10]. Mixed colonisation of the same niche by two species may provide conditions for genetic exchange between species.

Probing with a house-keeping gene (16S rDNA) and a Helicobacter species-specific urease gene revealed significant differences between $H$. bizzozeronii and $H$. salomonis in the localisation of these genes. Each gene was located in a different fragment in each $H$. bizzozeronii strain, whereas localisation in $H$. salomonis strains was homogeneous. Variability in gene order, i.e., macro-diversity, is typical of the $H$. pylori genome and, in this characteristic, the $H$. bizzozeronii genome may resemble the genome of $H$. pylori [10]. The macro-diversity of $H$. salomonis is perhaps less than that of $H$. pylori. More studies on gene order are needed. However, recently, genome mapping of $H$. bizzozeronii and $H$. salomonis has been restricted by the fact that except for 16S rRNA genes [3], sequences of other genes of these species are unknown. As in $H$. pylori [9], one urease gene was found in both organisms. Both organisms are urease positive [3]. The two $16 \mathrm{~S}$ ribosomal genes seen in $H$. bizzozeronii and $H$. salomonis equals the number seen in $H$. pylori $[8,10]$. The number of $23 \mathrm{~S}$ ribosomal genes was not determined, but bacteria usually have the same number for both genes [24]. The enterobacteria Escherichia coli and salmonellae usually have seven $16 \mathrm{~S}-23 \mathrm{~S}$ ribosomal genes [24]. A high number of copies is associated with the short generation time of fast-growing organisms, and a low number with the long generation time of slow-growing organisms - a characteristic typical of $H$. bizzozeronii [2], $H$. salomonis [3] and H. pylori [25].

The genome sizes of $H$. bizzozeronii and $H$. salomonis were in the range $c .1 .6-1.9 \mathrm{Mb}$ and $1.7-1.8 \mathrm{Mb}$, respectively. Similar sizes have been reported for the $H$. pylori genome, 1.6-1.7 [9, 10], while the genome size of $H$. hepaticus was estimated to be $1.3 \mathrm{Mb}$ [18]. The genetically related species Campylobacter jejuni and $C$. coli also have a genome of size of $1.7 \mathrm{Mb}$ [26], whereas E. coli and Salmonella spp. have genome sizes of $4.6 \mathrm{Mb}$ [24]. Genome size estimation of a larger number of species of phylogenetically related Helicobacter and Campylobacter spp. [1] will make it possible to judge whether small genome size is typical of this group of organisms. Small genome size is characteristic of bacterial species that are metabolically inactive $[10,24]$, such as $H$. bizzozeronii and $H$. salomonis [2, 3].

Not I and NruI are the enzymes most commonly used to study micro- and macro-heterogeneity of $H$. pylori $[9,10]$. Only Not I was useful for $H$. bizzozeronii and $H$. salomonis, and even Not I failed to cleave the DNA of one-third of $H$. bizzozeronii strains. The other rarecutters tested, PacI and SfII, either did not cut DNA or produced partial digestion, especially with $H$. bizzozeronii. These results, as well as results obtained with a frequently cutting enzyme Hae III which also does not cleave the DNA of many $H$. bizzozeronii strains (unpublished observations), suggest that methylation sites are common in the DNA of $H$. bizzozeronii. Because the $\mathrm{G}+\mathrm{C}$ mol \% content of $H$. bizzozeronii and $H$. salomonis is c. 42-43 (unpublished observations), Not I, Sfi I and $A s c \mathrm{I}$ as eightbasecutters with GC-rich sequences were more applicable than enzymes which recognise AT-rich sequences for digestion of $H$. bizzozeronii and $H$. salomonis DNA. However, PacI (TTAATTAA), which cleaves DNA rich in AT sequences, was useful for digestion of $H$. salomonis DNA.

In conclusion, macro-restriction analysis revealed significant genetic heterogeneity for $H$. bizzozeronii compared with homogeneity for $H$. salomonis. Both have a small genome and two $16 \mathrm{~S}$ ribosomal genes, characteristics typical of organisms that are metabolically inactive and have a slow growth rate.

The Finnish Academy of Science is acknowledged for financial support.

\section{References}

1. Paster BL, Lee A, Fox JG et al. Phylogeny of Helicobacter felis sp. nov., Helicobacter mustelae, and related bacteria. Int $J$ Syst Bacteriol 1991; 41: 31-38.

2. Hänninen M-L, Happonen I, Saari S, Jalava K. Culture and characteristics of Helicobacter bizzozeronii, a new canine gastric Helicobacter sp. Int $J$ Syst Bacteriol 1996; 46: $160-166$.

3. Jalava K, Kaartinen M, Utriainen M, Happonen I, Hänninen ML. Helicobacter salomonis sp. nov., a canine gastric Helicobacter sp. related to Helicobacter felis and Helicobacter bizzozeronii. Int J Syst Bacteriol 1997; 47: 975-982.

4. Eaton KA, Dewhirst FE, Paster BJ et al. Prevalence and varieties of Helicobacter species in dogs from random sources and pet dogs: animal and public health implications. $J$ Clin Microbiol 1996; 34: 3165-3170.

5. Hermanns WK, Kregel W, Breuer W, Lechner J. Helicobacterlike organisms: histopathological examination of gastric biopsies from dogs and cats. J Comp Pathol 1995; 112: 307-318.

6. Happonen I, Saari S, Castren L, Tyni O, Hänninen M-L, Westermarck E. Occurrence and topographical mapping of gastric Helicobacter-like organisms and their association with histological changes in apparently healthy dogs and cats. Zentralblatt Veterinarmed 1996; 43: 305-315.

7. Blaser MJ, Parsonnet J. Parasitism by the 'slow' bacterium Helicobacter pylori leads to altered gastric homeostasis and neoplasia. J Clin Invest 1994; 94: 4-8.

8. Tomb J-F, White O, Kerlavage AR et al. The complete genome sequence of the gastric pathogen Helicobacter pylori. Nature 1997; 388: 539-547.

9. Taylor DE, Eaton M, Chang N, Salama SM. Construction of a 
Helicobacter pylori genome map and demonstration of diversity at the genome level. $J$ Bacteriol 1992; 174: $6800-6806$.

10. Jiang Q, Hiratsuka K, Taylor DE. Variability of gene order in different Helicobacter pylori strains contributes to genome diversity. Mol Microbiol 1996; 20: 833-842.

11. Go MF, Kapur V, Graham DY, Musser JM. Population genetic analysis of Helicobacter pylori by multilocus enzyme electrophoresis: extensive allelic diversity and recombinational population structure. $J$ Bacteriol 1996; 178: 3934-3938.

12. Owen RJ, Hunton C, Bickely J, Moreno M, Linton D. Ribosomal RNA gene restriction patterns of Helicobacter pylori: analysis and appraisal of HaelII digests as a molecular typing system. Epidemiol Infect 1992; 109: 35-47.

13. Akopyanz N, Bukanov NO, Westblom TU, Kresovich S, Berg DE. DNA diversity among clinical isolates of Helicobacter pylori detected by PCR-based RAPD fingerprinting. Nucleic Acids Res 1992; 20: 5137-5142.

14. Desai M, Linton D, Owen RJ, Stanley J. Molecular typing of Helicobacter pylori isolates from asymptomatic, ulcer and gastritis patients by urease gene polymorphism. Epidemiol Infect 1994; 112: $151-160$.

15. Fujimoto S, Marshall B, Blaser MJ. PCR-based restriction fragment length polymorphism typing of Helicobacter pylori. $J$ Clin Microbiol 1994; 32: 331-334.

16. Cover TL, Tummuru MKR, Cao P, Thomson SA, Blaser MJ Divergence of genetic sequences for the vacuolating cytotoxin among Helicobacter pylori strains. J Biol Chem 1994; 269 : 10566-10573.

17. Taylor DE, Chang N, Taylor NS, Fox JG. Genome conservation in Helicobacter mustelae as determined by pulsed-field gel electrophoresis. FEMS Microbiol Lett 1994; 118: 31-36.

18. Saunders KE, McGovern KJ, Fox JG. Use of pulsed-field gel electrophoresis to determine genomic diversity in strains of Helicobacter hepaticus from geographically distant locations. $J$ Clin Microbiol 1997; 35: 2859-2863.

19. Gibson JR, Sutherland K, Owen RJ. Inhibition of DNase activity in PFGE analysis of DNA from Campylobacter jejuni. Lett Appl Microbiol 1994; 19: 357-358.

20. Hänninen M-L, Hirvelä-Koski V. Pulsed-field gel electrophoresis in the study of mesophilic and psychrophilic Aeromonas spp. J Appl Bacteriol 1997; 83: 493-498.

21. Riley LK, Franklin CL, Hook RR, Besch-Williford C. Identification of murine helicobacters by PCR and restriction enzyme analysis. J Clin Microbiol 1996; 34: 942-946.

22. Solnick JV, O'Rouke J, Lee A, Tomkins LS. Molecular analysis of urease genes from a newly identified uncultured species of Helicobacter. Infect Immun 1994; 62: 1631-1638.

23. Lockard VG, Boler RK. Ultrastructure of a spiraled microorganism in the gastric mucosa of dogs. Am J Vet Res 1970; 31: $1453-1462$.

24. Cole ST, Saint Girons I. Bacterial genomics. FEMS Microbiol Rev 1994; 14: 139-160.

25. Goodwin CS, Amstrong JA. Microbiological aspects of Helicobacter pylori (Campylobacter pylori). Eur J Clin Microbiol Infect Dis 1990; 9: 1-13.

26. Chang N, Taylor DE. Use of pulsed-field agarose gel electrophoresis to size genomes of Campylobacter species and to construct a SalI map of Campylobacter jejuni UA580. $J$ Bacteriol 1990; 172: 5211-5217. 